\title{
Assessment of air pockets in high-dose-rate vaginal cuff brachytherapy using cylindrical applicators
}

\author{
Ashraf Hassouna, MD',2, Prof. Yasir Abdulaziz Bahadur³, Camelia Constantinescu, PhD' \\ IKing Faisal Specialist Hospital \& Research Center, Jeddah, Saudi Arabia, ${ }^{2}$ National Cancer Institute, Cairo University, Egypt, ${ }^{3}$ King Abdulaziz \\ University Hospital, Jeddah, Saudi Arabia
}

\begin{abstract}
Purpose: To retrospectively assess the incidence and magnitude of air pockets around vaginal cylinders and its impact on dose distribution in vaginal cuff image-guided high-dose-rate (HDR) brachytherapy.

Material and methods: Fifty endometrial carcinoma patients treated by postoperative HDR vaginal cuff brachytherapy were included in the study. The average age of patients was $58.3 \pm 11.8$ years (range: $31-87$ years). Brachytherapy was performed using cylindrical applicators, and the dose prescribed to $0.5 \mathrm{~cm}$ from the applicator's surface, over a length of $5 \mathrm{~cm}$ from the applicator's tip. Computed tomography (CT) simulation was used for each brachytherapy fraction. The incidence, vaginal mucosa displacement, volume, and dosimetric effect of air pockets around the vaginal cylinder were evaluated.

Results: A total of 78 air pockets were found in $29 / 50$ patients (58\%) and 45/135 (33\%) brachytherapy plans. They were located at the apex: $16 / 78(20 \%)$ and lateral to the applicator: $62 / 78(80 \%)$. The volume of air pockets ranged between 0.01 and $2.1 \mathrm{~cm}^{3}$ (mean: $0.15 \mathrm{~cm}^{3} \pm 0.36 \mathrm{~cm}^{3}$ ), and the maximum displacement of vaginal mucosa from cylinder surface was between 0.1 and $1.09 \mathrm{~cm}$ (mean: $0.34 \mathrm{~cm} \pm 0.2 \mathrm{~cm}$ ). The dose reduction to the vaginal mucosa generated by the air pockets ranged from 0.5 to $66 \%$ (mean: $26.4 \% \pm 13.9 \%$ ).

Conclusions: The presence of air pockets around vaginal cylinder applicators is frequently noticed in post-operative vaginal cuff brachytherapy. The dose to the vaginal mucosa is reduced, as a result of displacement generated by air pockets. The effect on the clinical outcome of this dose reduction is yet to be determined.
\end{abstract}

J Contemp Brachytherapy 2014; 6, 3: 27l-275 DOI: $10.5114 /$ jcb.2014.45436

Key words: air pockets, brachytherapy cylinder, endometrial carcinoma, vaginal brachytherapy.

\section{Purpose}

Post-operative vaginal cuff brachytherapy is used to reduce the risk of vault recurrence for patients with endometrial or cervical carcinomas [1,2]. Although the choice of applicator for high-dose-rate (HDR) brachytherapy of vagina is both institutional and patient dependent, the most commonly used applicator is a vaginal cylinder [1]. Recently, the American Brachytherapy Society (ABS) reported the recommendations for adjuvant vaginal HDR brachytherapy after hysterectomy, establishing dose prescription and optimization guidelines [3]. As the prevalent location of the vaginal lymphatic channels is within $1 \mathrm{~mm}$ of tissue surrounding the vaginal cylinder, the ABS report recognizes that it is imperative for the vaginal mucosa to be in contact with the cylindrical applicator surface to achieve the optimal dose distribution, and recommends computed tomography (CT) planning to confirm the presence of significant air gaps [3,4]. Air pockets around vaginal cylinders have been often noticed in our brachytherapy practice. Their presence may result in the failure to eradicate all microscopic malignant cells, therefore increasing the risk of recurrence.

To date, there is not enough literature addressing this subject $[5,6]$. The purpose of this study is to retrospectively assess the incidence and magnitude of air pockets around vaginal cylinders, and its impact on dose distribution in CT-image based HDR brachytherapy.

\section{Material and methods}

We retrospectively reviewed the data of 50 patients with endometrial carcinoma, treated between July 2010 and September 2013 by post-operative HDR vaginal cuff brachytherapy in the Radiotherapy Unit of King Abdulaziz University Hospital Jeddah, Saudi Arabia. The patients had either post-operative external beam radiotherapy (EBRT; 45 Gy in 25 fractions, one fraction per day, five times per week) to the whole pelvis, using four-fields CTbased planning, followed by HDR brachytherapy (12 Gy in 3 fractions, two fractions per week), or HDR brachytherapy alone (21 Gy in 3 fractions, one fraction per week).

Address for correspondence: Yasir A. Bahadur, MD, Department of Radiology, King Abdulaziz University Received: 06.05.2014 Hospital, Jeddah, Saudi Arabia, PO Box 80215, Jeddah 21589, Saudi Arabia, phone: +966 505528528, 
Clinical examination was performed before brachytherapy, to assess the patient and estimate the applicator size to be used. The appropriate cylinder size was selected according to the clinical examination before brachytherapy. Clinical judgement (inserting the cylinder according to the vaginal stump length and monitoring patient discomfort during insertion) was used to insure that the cylinder reaches the vaginal apex. Gold seeds were not used to mark the vaginal apex. Application was completed without anesthesia, and the applicator was fixed in place by a perineal belt ensuring its immobilization. Brachytherapy was carried out with cylindrical vaginal applicators of various diameters $(2,2.6,3$, and $3.5 \mathrm{~cm})$, and the dose prescribed to $0.5 \mathrm{~cm}$ from the applicator's surface, over a length of $5 \mathrm{~cm}$ from the applicator's tip.

Computed tomography images were acquired for each brachytherapy fraction using a Siemens Somatom Emotion CT scanner (Siemens AG, Erlangen, Germany), with $2 \mathrm{~mm}$ slice intervals from the iliac crest to the ischial tuberosities, without intravenous contrast. Dose distribution was calculated by Varian Brachyvision planning system, version 8.10 (Varian Medical Systems, Inc., Palo Alto, CA), for a brachytherapy remote afterloader Varian HDR VariSource iX (Varian Medical Systems, Inc.). The dose cal- culation algorithm is based on the TG-43 formalism, as recommended by the American Association of Physicists in Medicine (AAPM) [7]. All treatment plans were retrospectively analyzed for the presence of air pockets in the proximal $5 \mathrm{~cm}$ of the vaginal vault, that were contoured, and the volumes calculated by the treatment planning system, as shown in Figure 1.

The incidence, vaginal mucosa displacement, volume, and dosimetric effect of the air pockets around the vaginal cylinder were evaluated.

\section{Results}

For 50 patients, a total number of 135 CT-based brachytherapy plans were retrospectively reviewed. The average age of patients was $58.3 \pm 11.8$ years (range: 31 87 years). We could not find any correlation between the incidence of air pockets and patient's age. A total of 78 air pockets were found in $29 / 50$ patients (58\%) and 45/135 (33\%) brachytherapy plans. They were located at the apex: 16/78 (20\%) and lateral to the applicator: $62 / 78(80 \%)$. The apex air pockets occurred during first brachytherapy fraction for 4 patients and in subsequent fractions for 6 patients. One patient presented air pockets at applicator apex through all treatment.

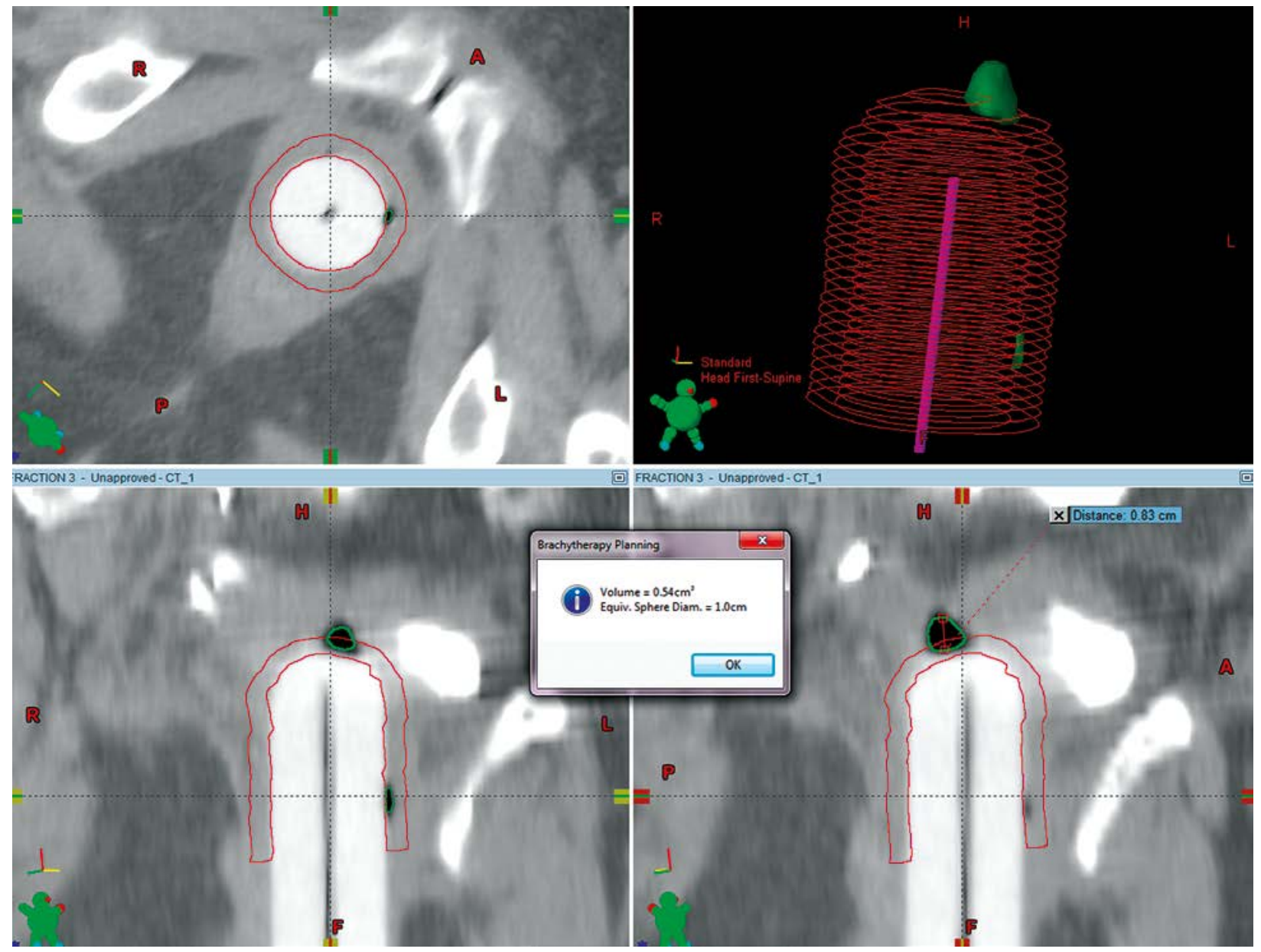

Fig. 1. Computed tomography images of air pockets present around a vaginal cylinder for a representative patient, in axial and sagittal views 


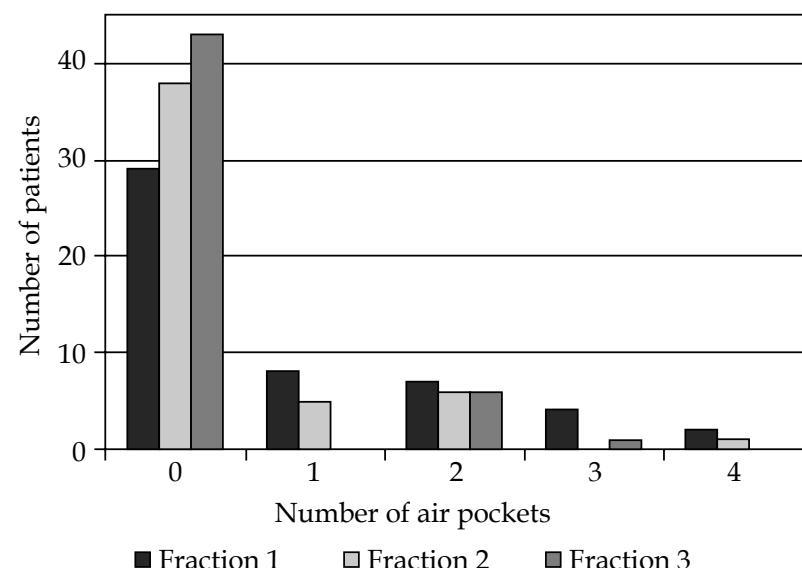

Fig. 2. Number of air pockets per patient over the course of 3 brachytherapy fractions

The incidence of air pockets per patient during the course of 3 brachytherapy fractions is presented in Figure 2. Among the 29 patients presenting air pockets, 21 (72\%) had them occurring during first fraction of treatment, while $8(28 \%)$ patients developed air pockets later in their brachytherapy course: 6 (21\%) of them during second fraction and $2(7 \%)$ during third fraction. Three patients presented air pockets during all treatment. The maximum number of air pockets for a single patient during the whole brachytherapy course was 8 .

The correlation between the incidence of air pockets and vaginal applicator size was analysed and is shown in Figure 3. The highest incidence of air pockets occurred for $3 \mathrm{~cm}$ cylinder diameter, in 25/48 (52\%) fractions, and the lowest for 2 and $2.6 \mathrm{~cm}$ diameter, in $7 / 9(21 \%)$ and $7 / 11$ $(20 \%)$ fractions, respectively.

The volume of air pockets ranged between 0.01 and $2.1 \mathrm{~cm}^{3}$ (mean: $0.15 \mathrm{~cm}^{3} \pm 0.36 \mathrm{~cm}^{3}$ ) and the maximum displacement of vaginal mucosa from cylinder surface was between 0.1 and $1.09 \mathrm{~cm}$ (mean: $0.34 \mathrm{~cm} \pm 0.2 \mathrm{~cm}$ ). The dosimetric impact of air pockets was the reduction of dose to the vaginal mucosa from $0.5 \%$ to $66 \%$ (mean: $26.4 \% \pm 13.9 \%$ ), as presented in Figure 4. Even for small displacements of vaginal wall $(0.1-0.2 \mathrm{~cm})$, the dose reduction exceeds $10 \%$. The dose reduction is increasing with the air pockets displacement, this effect being more significant for cylinders with small diameters due to the inverse square factor.

\section{Discussion}

Adapting a HDR vaginal brachytherapy plan to patient specific anatomy is challenging, as recognized by the recent ABS guidelines for adjuvant vaginal cuff brachytherapy after hysterectomy [3]. The applicator selection may be influenced by the post-operative shape of vagina, but is mainly physician or institutional depending, and the most commonly used applicator is a properly sized vaginal cylinder [8]. Regardless the choice of applicator, and though the total dose required to eradicate the disease is unknown, it is essential for the vaginal mucosa to be in contact with the applicator surface to achieve the optimal dose distribution $[3,9]$.

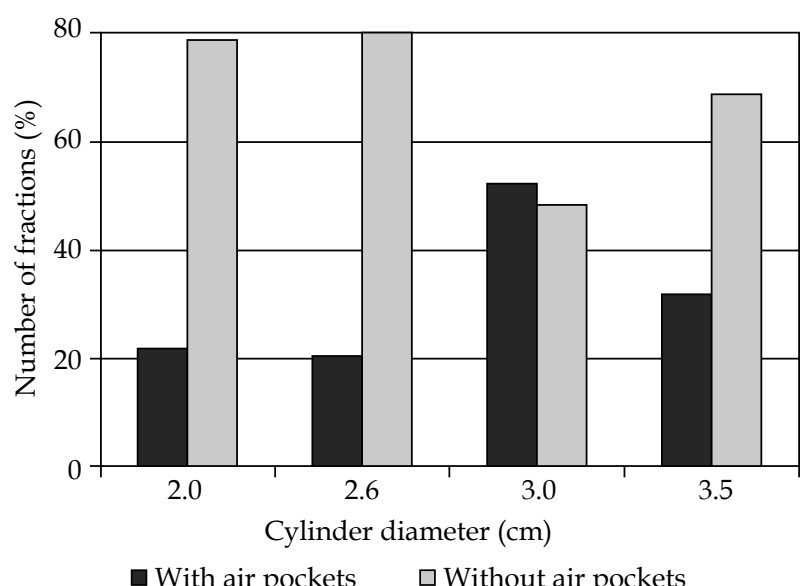

Fig. 3. The distribution of air pockets according to vaginal cylinder diameter

The vaginal cylinder should be fitted to the vagina in size and introduced to reach the vaginal vault. Richardson $e t$ al. used gold seeds to mark the vaginal vault apex and CT-imaging to verify that the vaginal cylinder reached these seeds [5]. In our study, we have used the clinical sense and patient's feeling to ensure that the cylinder reached the vaginal vault apex. We reported $58 \%$ incidence of air gaps in our patients and 16/78 (20\%) being at the apex, while Richardson et al. [5] reported $80 \%$ incidence of air gaps for all patients and 21/90 (23\%) located at the apex. It seems that clinical judgement works well and gold seeds are not mandatory to verify the cylinder-vaginal vault relationship.

In some patients, the vagina may not be cylindrical after surgery and have an enlargement in the lateral apices, due to surgical residues of the vaginal fornices. Parity, hormonal status, or normal anatomic irregularities (e.g., larger vaginal apex in relation to introitus) can make difficult the adequate placement of a vaginal cylinder. Also,

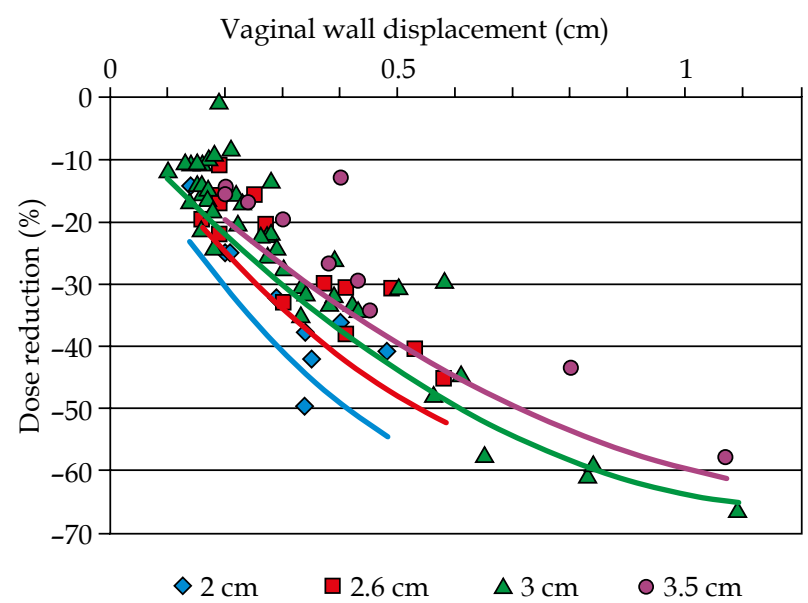

Fig. 4. The percentage dose differences between cylinder lateral surface and vaginal mucosa surface at the point of maximum displacement created by the air pockets, for various diameters of vaginal cylinders. The solid trend lines represent the inverse square factor for each cylinder 
if the chosen cylinder is too small, air gaps or folds may be present, leading to under-dosage of the vaginal target tissue. Alternative approaches for a treatment individualized to the patient's anatomy are the use of ovoids for vaginas with "dog-ear" configuration, or a vaginal custom mold $[10,11]$. The use of anatomically conformal applicators, such as intra-vaginal balloon applicator or multi-channel applicator has been also suggested $[12,13]$.

Vaginal relapse rates in post-operative vaginal cuff brachytherapy for endometrial carcinoma patients are reported as ranging from less than $5 \%$ to $18 \%$ [1,14-16]. Since the predominant location of the vaginal lymphatic channels is within $1 \mathrm{~mm}$ of tissue surrounding the vaginal cylinder [4], a possible cause of relapse is that the target volume does not receive the prescribed dose, due to the presence of air pockets. Besides, many institutions do not perform fractional imaging of brachytherapy treatments; therefore the size and significance of these air pockets are widely undetermined.

We found 78 air pockets in 29/50 patients (58\%) and $45 / 135$ (33\%) brachytherapy plans. Out of 50 patients, $29(58 \%)$ did not reveal air pockets during the first fraction of treatment, but had air pockets present in following fractions, and $43(86 \%)$ patients had no air pockets during last fraction of treatment. This indicates that CT-imaging only the first fraction of brachytherapy is insufficient for assessing the conformity of vaginal mucosa to the cylinder over the whole course of treatment, as suggested by other investigators $[5,17]$ as well. Yaparpalvi et al. analysed the inter-fraction variations of cylindrical applicator insertion, as well as the fluctuations in bladder and rectal volumes, which have led to variations of bladder and rectal doses; they concluded that each fraction of vaginal cuff brachytherapy should be image-based, in order to achieve an accurate and complete dosimetric assessment of the treatment [17].

Analysing the correlation between the incidence of air pockets and vaginal applicator size, we found that the highest incidence of air pockets occurred for $3 \mathrm{~cm}$ cylinder diameter, and the lowest for 2 and $2.6 \mathrm{~cm}$ diameter. Although selecting the largest tolerated applicator was suggested to ensure an optimal contact with the vaginal mucosa, it is not necessary to be associated with fewer incidences of air pockets. A prospective study is necessary to explore the role of using larger applicator in the remaining fractions in patients having air pockets in the first brachytherapy application.

Our data show that, even for small displacements of vaginal wall $(1-2 \mathrm{~mm})$, the dose reduction exceeds $10 \%$. The dose reduction is increasing with the displacement generated by air pockets, this effect being more significant for cylinders with small diameters due to the inverse square factor.

Similar data have been reported by Richardson et al. [5]. In a study analysing brachytherapy plans of 25 patients with post-operative endometrial carcinoma, they found an average pocket volume of $0.34 \mathrm{~cm}^{3}$ (range: $0.01-1.32 \mathrm{~cm}^{3}$ ), and an average mucosa displacement of $0.37 \mathrm{~mm}$ (range: $0.13-0.8 \mathrm{~mm}$ ), the dosimetric impact being a reduction of the dose to the vaginal mucosa of about $27 \%$ (range: $9-58 \%$ ). Cameron et al. compared the dose to $0.5 \mathrm{~cm}$ from the surface of the vaginal mucosa at air gaps location to the dose that mucosa would have received if there was no air gap, and found it ranging between $54.7 \%$ and $97.3 \%$ (average: $86.7 \%$ ) [6]. Although barely reported, the presence of air pockets around vaginal cylinders seems to be a frequent event in vaginal cuff brachytherapy. However, brachytherapy appears to reduce the risk of vaginal relapse despite the vaginal mucosa potentially being displaced from the applicator by air pockets $[1,14-16,18]$.

Even if air pockets have a considerable dosimetric effect, it seems that it may be of minor clinical consequence. The highest risk of microscopic malignant cells prevails for vaginal mucosa located at the apex of applicator. Our data show that only $20 \%$ of air pockets were located at the apex, this finding decreasing the risk of not treating the microscopic disease. Moreover, except for one patient, the position of air gaps was different for each brachytherapy fraction, additionally attenuating their overall effect, as also noticed by Cameron et al. [6].

Furthermore, for the most treatment planning systems currently available, the brachytherapy dose calculation algorithms do not take in account the tissue inhomogeneity, and the dose is calculated as if the sources are surrounded by water. Yet, if low density air pockets are present, the delivered doses are fairly increased by scattering effects from adjacent tissues. The literature indicates that post-hysterectomy vaginal HDR brachytherapy is an efficient treatment, resulting in good clinical outcome for many different dose and fractionation schedules [3]. However, the dose required to eradicate microscopic disease is still unknown. Randomizing 290 patients with low-risk endometrial carcinoma to 15 vs. 30 Gy in 6 fractions, prescribed to $0.5 \mathrm{~cm}$ vaginal tissue, Sorbe et al. reported no difference in local recurrence [9]. It is not yet established if this is valid for high-intermediate-risk disease too, but it is possible that a lower dose would be similarly efficient. Therefore, even if air pockets are present, the dose to vaginal mucosa may still be sufficient to treat microscopic malignant cells [6].

The magnitude of air pockets around vaginal cylinders and their effect on dose distribution was scarcely reported in the literature $[5,6]$, and our report will add more evidence that this issue substantiate attention and more research. Further clinical trials are needed to estimate the uncertainties in delivering vaginal cuff HDR brachytherapy $[18,19]$.

\section{Disclosure}

Authors report no conflict of interest.

\section{References}

1. Horowitz NS, Peters WA 3rd, Smith MR et al. Adjuvant high dose rate vaginal brachytherapy as treatment of stage I and II endometrial carcinoma. Obstet Gynecol 2002; 99: 235-240.

2. Pearcey RG, Petereit DG. Post-operative high dose rate brachytherapy in patients with low to intermediate risk endometrial cancer. Radiother Oncol 2000; 56: 17-22.

3. Small W, Beriwal S, Demanes DJ et al. American Brachytherapy Society consensus guidelines for adjuvant vaginal cuff 
brachytherapy after hysterectomy. Brachytherapy 2012; 11: 58-67.

4. Choo JJ, Scudiere J, Bitterman P et al. Vaginal lymphatic channel location and its implication for intracavitary brachytherapy radiation treatment. Brachytherapy 2005; 4: 236-240.

5. Richardson S, Palaniswaamy G, Grigsby PW. Dosimetric effects of air pockets around high-dose-rate brachytherapy vaginal cylinders. Int J Radiat Oncol Biol Phys 2010; 78: 276-279.

6. Cameron AL, Cornes P, Al-Booz H. Brachytherapy in endometrial cancer: Quantification of air gaps around a vaginal cylinder. Brachytherapy 2008; 7: 355-358.

7. Rivard MJ, Coursey BM, DeWerd LA et al. Update of AAPM Task Group No. 43 Report. A revised AAPM protocol for Brachytherapy dose calculations. Med Phys 2004; 31: 633-674.

8. Small JW, Erickson B, Kwakwa F. American Brachytherapy Society survey regarding practice patterns of postoperative irradiation for endometrial cancer: Current status of vaginal brachytherapy. Int J Radiat Oncol Biol Phys 2005; 63: 1502-1507.

9. Sorbe B, Straumits A, Karlsson L. Intravaginal high-dose-rate brachytherapy for stage I endometrial cancer: A randomized study of two dose-per-fraction levels. Int J Radiat Oncol Biol Phys 2005; 62: 1385-1389.

10. Tuncel N, Garipagaoglu M, Kizildag AU et al. Optimisation techniques in vaginal cuff brachytherapy. Br J Radiol 2009; 82: 936-940.

11. Pötter R, Gerbaulet A, Haie-Meder C. The GEC ESTRO handbook of brachytherapy. Endometrial Cancer 2002; 15: 365-401.

12. Miller DA, Richardson S, Grigsby PW. A new method of anatomically conformal vaginal cuff HDR brachytherapy. Gynecol Oncol 2010; 116: 413-418.

13. Symon Z, Menhel J, Alezra D et al. Individual fraction optimization vs. first fraction optimization for multichannel applicator vaginal cuff high-dose-rate brachytherapy. Brachytherapy 2006; 5: 211-215.

14. Knocke TH, Kucera H, Weidinger B et al. Primary treatment of endometrial carcinoma with high-dose-rate brachytherapy: Results of 12 years of experience with 280 patients. Int I Radiat Oncol Biol Phys 1997; 37: 359-365.

15. MacLeod C, Fowler A, Duval P et al. High-dose-rate brachytherapy alone post-hysterectomy for endometrial cancer. Int J Radiat Oncol Biol Phys 1998; 42: 1033-1039.

16. Alektiar KM, Venkatraman E, Barakat RR. Intravaginal brachytherapy alone for intermediate-risk endometrial cancer. Int J Radiat Oncol Biol Phys 2005; 62: 111-117.

17. Yaparpalvi R, Mutyala S, Thawani N et al. Variance in bladder and rectal doses in the course of fractionated cylinder high-dose-rate brachytherapy. Brachytherapy 2009; 8: 145.

18. Nout RA, Smit VT, Putter H et al. Vaginal brachytherapy versus pelvic external beam radiotherapy for patients with endometrial cancer of high-intermediate risk (PORTEC-2) an open-label, non-inferiority, randomised trial. Lancet 2010; 375: 816-823.

19. Reed NS, Harrand RL. Counterpoint: The role of adjuvant radiation in endometrial cancer. Inside, outside, or not at all? Brachytherapy 2011; 10: 4-6. 